\title{
Does a pear growl? Interference from semantic properties of orthographic neighbors
}

\author{
Diane Pecher, Jimmy de RooiJ, And René Zeelenberg \\ Erasmus University, Rotterdam, The Netherlands
}

\begin{abstract}
In this study, we investigated whether semantic properties of a word's orthographic neighbors are activated during visual word recognition. In two experiments, words were presented with a property that was not true for the word itself. We manipulated whether the property was true for an orthographic neighbor of the word. Our results showed that rejection of the property was slower and less accurate when the property was true for a neighbor than when the property was not true for a neighbor. These findings indicate that semantic information is activated before orthographic processing is finished. The present results are problematic for the links model (Forster, 2006; Forster \& Hector, 2002) that was recently proposed in order to bring form-first models of visual word recognition into line with previously reported findings (Forster \& Hector, 2002; Pecher, Zeelenberg, \& Wagenmakers, 2005; Rodd, 2004).
\end{abstract}

Researchers have been interested for quite some time now in how reading a word proceeds from the initial processing of its visual features to the activation of its meaning. Various models have been proposed in order to account for semantic processing of visually presented words. Strictly form-first models propose that a word's semantic properties are activated only after orthographic processing has completed and a unique word has been identified and selected for further processing (Forster, 2006; Forster \& Hector, 2002). In these models, presentation of a target word activates not only the orthographic representation of the target word itself, but also that of orthographic neighbors.

Processing at the orthographic level continues until a unique word has been identified. Only then is information from this level used as input for processing at the semantic level. Cascaded models (see, e.g., Becker, Moscovitch, Behrmann, \& Joordens, 1997; Masson, 1995; McClelland \& Rumelhart, 1981), on the other hand, assume that information cascades from one level to the next as soon as processing has started. In these models, too, presentation of a target word activates the orthographic representation of the target word itself, as well as that of orthographic neighbors. However, in contrast to form-first models, as soon as a word's orthographic representation starts being activated, information from the orthographic level is used as input for other levels. Thus, semantic information gets activated before the processing of orthography has been completed. ${ }^{1}$ The distinction between form-first and cascaded activation is fundamental to models of word recognition. For example, cascaded activation is a necessary condition for certain effects of feedback processing on visual word recognition (Hino, Lupker, \& Pexman, 2002; Pecher, 2001; Pexman \& Lupker, 1999; Pexman, Lupker, \& Hino, 2002; Stone, Vanhoy, \& Van Orden, 1997; Van Orden \& Goldinger, 1994; Ziegler, Montant, \& Jacobs, 1997).

Evidence for cascaded processing is provided by studies that show that semantic properties of a target's neighbors affect responding to the target word itself (Bourassa \& Besner, 1998; Duñabeitia, Carreiras, \& Perea, 2008; Forster \& Hector, 2002; Pecher, Zeelenberg, \& Wagenmakers, 2005). Forster and Hector presented words and nonwords in an animal decision task. They observed that categorization was slower for nonwords (e.g., turple) that had an animal neighbor (e.g., turtle) than for nonwords (e.g., cishop) that did not have an animal neighbor. These results are consistent with the view that semantic properties of the neighbor are activated before the target is recognized as being a nonword (but see below for an alternative interpretation). Had orthographic processing been completed before the activation of the semantic information, the semantic properties of the neighbor would not have interfered with the decision.

An important characteristic of the Forster and Hector (2002) study is that the critical manipulation was done with nonword stimuli. Because nonwords do not have lexical representations, it is possible that the word recognition system settled on the lexical representation of the word most similar to the nonword. Different results might therefore be obtained when the critical stimuli are words. To investigate whether semantic properties of

D.Pecher, pecher@fsw.eur.nl 
neighbors are activated during the processing of words, Rodd (2004) and Pecher et al. (2005) presented word targets in semantic decision tasks. Both studies showed better performance for words (e.g., monkey) that had (mostly) congruent neighbors (i.e., orthographic neighbors from the same task-relevant category, such as donkey) than for words (e.g., gibbon) that had (mostly) incongruent neighbors (i.e., neighbors from the opposite category, such as ribbon). Similar effects were obtained with subset stimuli (e.g., target $=$ paper , neighbor $=$ ape $)$ and superset stimuli (e.g., target $=$ hark, neighbor $=$ shark $)($ Bowers, Davis, \& Hanley, 2005).

These findings provide evidence for cascaded models of word processing and are problematic for strictly formfirst models. In order to save form-first models, Forster and Hector (2002; see also Forster, 2006) offered the links model as an alternative explanation of their results. The links model assumes that lexical entries contain links to semantic fields. These semantic fields correspond roughly to taxonomic categories or to other types of clusterings that are based on lexical co-occurrence. Two examples of semantic fields are fruits and vegetables and animals. The links are crude semantic indices that have no meaning themselves and are assumed to be available at early stages of orthographic processing. ${ }^{2}$ During the initial stage of processing, several lexical items that are orthographically similar to the input are activated and treated as candidate lexical items. In a category decision task, such as animal decision, candidate lexical items are evaluated for whether they have the appropriate link. The verification process bypasses candidates that do not have the appropriate link. If, however, a candidate contains a link to the relevant semantic field (e.g., the animal field), the verification process cannot bypass it. Candidates that do have the appropriate link require verification. This mechanism can explain the results (Forster \& Hector, 2002; Pecher et al., 2005; Rodd, 2004 ) that have shown impaired performance to nonexemplar targets (e.g., an inanimate word, such as noodle) that have an exemplar neighbor (e.g., an animate word, such as poodle). In such cases, the exemplar neighbor has a link to the animal field. When this link is detected, the neighbor is treated as a candidate, and its lexical entry has to be verified against the input before a decision is made. This additional verification takes time and causes a slowdown in responding to the target.

According to the links model, the semantic congruency of neighbors should affect processing only in tasks that require access to existing categories, such as animal, but not for other types of semantic processing. Thus, the links model can explain an effect of exemplar neighbors on nonexemplars in animacy decision because links to an animal field slow down processing. Also consistent with the links model, Forster (2006) obtained no effect of exemplar neighbors in a physical object decision task. The links model can explain these results because there is no semantic field for the physical object category. Contrary to Forster's results, however, Boot and Pecher (2008; see also Pecher et al., 2005, Experiment 4) found congruency effects in a size decision task (Is it larger than a shoebox?). The links model predicts no effect of neighbor congru- ency in size decision, because size (in particular, things larger than a shoebox) is not a semantic field (Forster, 2006). Thus, at present, the evidence for the links model is mixed.

To further test the links model, we used a property verification task in the present study. In this task, on each trial, a target word was presented with a property (e.g., A pear can growl), and the task was to decide whether the property was true for the target word. This approach has several advantages. First, properties such as can growl or is made of metal do not form existing categories (i.e., these properties do not correspond to semantic fields). Therefore, evidence that specific semantic properties of a target's neighbors are activated would be problematic for the links model, but would be in line with cascaded models of visual word recognition. Second, we presented a different property on each trial, and the target word was presented before the property. Therefore, the system was not able to use this information to bypass the verification process for lexical candidates - a critical assumption that was used to account for the results obtained with the animal decision task. To test whether the semantic representations of neighbors would be activated, we used a property verification task in which participants verified properties that were false for the target word (which was always the name of an object) but true for a neighbor of the target (e.g., A pear can growl). If semantic properties (e.g., growl) of orthographic neighbors (e.g., bear) are available before the target word (e.g., pear) has been identified, then it should be harder to reject a semantic property of a neighbor than to reject a property that is not true for any neighbor of the target. ${ }^{3}$

\section{EXPERIMENT 1}

\section{Method}

Participants. Fifty-four students from Erasmus University Rotterdam participated for partial course credit. All of the participants were native Dutch speakers.

Stimuli. A set of 78 Dutch noun-property pairs was created. Each property was true for one of each noun's orthographic neighbors, but not for the target noun itself. For example, one of the pairs was biet-heeft een schuimkraag. The property heeft een schuimkraag (has a foamy head) is not true for biet (beet), but it is true for bier (beer), which is an orthographic neighbor of biet. For each targetneighbor pair, the neighbor's word frequency was higher than the target's. The noun-property pairs were presented in short sentences, such as Een biet heeft een schuimkraag (A beet has a foamy head). The pairs can be found at poseidon.eur.nl/memorylab/Publications/ StimuliNeighborProperty.pdf. An unrelated control condition was created by recombining noun-property pairs, so that the unrelated property was not true for the noun itself, nor for any of its orthographic neighbors (e.g., Een biet is van kraakbeen [A beet is made of cartilage]). Two counterbalanced lists were created, so that nouns and properties were counterbalanced across lists. Half of the experimental pairs on each list were related, and the other half were unrelated. Half of the participants received one list, and the other half received the other list. An additional set of 16 practice and 80 filler sentences was created. The filler sentences all required a true response (e.g., $A$ sweater has sleeves, A bottle can break). The practice set consisted of 8 true noun-property pairs and 8 false noun-property pairs.

Procedure. The participants were instructed to judge for each sentence whether the property was true or false for the noun. The sentences were presented on the computer screen in random order. 
Table 1

Mean Reaction Times (RTs, in Milliseconds),

Error Percentages (EPs), and Standard Errors (SEs)

in the Property Verification Task of Experiment 1

\begin{tabular}{|c|c|c|c|c|}
\hline \multirow[b]{2}{*}{ Condition } & \multicolumn{2}{|c|}{ RT } & \multicolumn{2}{|c|}{ EP } \\
\hline & $M$ & $S E$ & $M$ & $S E$ \\
\hline Unrelated & 1,161 & 28 & 7.4 & 1.1 \\
\hline Related & 1,191 & 31 & 9.4 & 0.9 \\
\hline Neighbor interference effect & 30 & 10 & 2.0 & 0.7 \\
\hline
\end{tabular}

Note-In the related condition, the property was true for a neighbor of the target noun. In the unrelated condition, the property was not true for any neighbor of the target noun.

A trial started with a fixation signal $(+)$ that was displayed for $250 \mathrm{msec}$ at the location where the first letter of the sentence was to appear. Then the first part of the sentence (containing the noun, but not the property; e.g., $A$ beet) was displayed for $50 \mathrm{msec}$. Subsequently, the rest of the sentence was added, so that the complete sentence ( $A$ beet has a foamy head) was displayed until the participant responded by pressing the " $m$ " key to indicate that the property was true or the " $z$ " key to indicate that the property was false. If the response was incorrect, the message "incorrect" was displayed for $1,500 \mathrm{msec}$. If the response exceeded the 2,500-msec deadline, the message "te laat" (too late) was displayed for 1,500 msec. Following the response or feedback, a 500-msec blank screen appeared, and then the next trial started. The experiment started with 16 practice trials, which were followed by 158 experimental and filler trials.

\section{Results}

For each condition, the mean reaction time (RT) was calculated. RTs to correct responses that fell outside three $S D$ s of the participant's mean were excluded from this calculation. This trimming procedure resulted in the removal of $1.4 \%$ of the RTs to correct responses. RTs for incorrect responses were also excluded. Table 1 shows the mean RTs for correct responses and the error percentages (EPs) for each condition. Statistical analyses were done with relatedness as a within-participants factor and list version as a between-participants factor. ${ }^{4}$ Responses were slower to properties that were true for a neighbor of the target than to properties that were not true for any of the neighbors of the target $[F(1,52)=8.44, p<.01] .^{5}$ Participants also made more errors to properties that were true for a neighbor of the target noun than to properties that were not true for any of the neighbors of the target noun $[F(1,52)=9.81, p<.01]$. Thus, performance was better when the property was not true for a neighbor than when it was true for a neighbor.

\section{EXPERIMENT 2}

Experiment 1 showed that rejecting a false property for a target noun was harder for properties that were true for an orthographic neighbor of the target (e.g., A pear can growl) than for properties that were not true for any neighbor of the target (e.g., A pear can make a web). Our explanation of this effect is that during orthographic processing of the target word, its orthographic neighbors and their semantic properties are activated. The activation of a property that is not true for the target word results in slower RTs and higher EPs.

The aim of Experiment 2 was to extend the findings of Experiment 1. In Experiment 1, we presented target words with properties that were quite specific to a particular neighbor. For example, meow was used for vat, a neighbor of cat. Meowing is a specific property of cats and is not shared by many other entities. Perhaps such specific and highly characteristic properties are more accessible than other properties. If this is the case, only those specific properties of neighbors may be activated. Consequently, the effects obtained in Experiment 1 may be restricted to the particular type of materials that we used. In Experiment 2, we investigated whether the results of Experiment 1 would extend to properties that are less specific than the ones used in Experiment 1. To this end, we used a different set of properties that were less specific because they were true for many entities - for example, the property can growl or is made of metal. The question of interest was whether, with these less specific properties, we would again find impaired performance for properties that are true for the neighbor but not for the target.

\section{Method}

Participants. Fifty-four students from Erasmus University Rotterdam participated for partial course credit. All of the participants were native Dutch speakers. None had participated in Experiment 1.

Stimuli and Procedure. The same set of target nouns and orthographic neighbors was used as in Experiment 1, except for two nouns that were replaced by new nouns. The target nouns were paired with a new set of properties. We selected properties that were less specific to their neighbors than those used in Experiment 1, such as can growl or is made of metal. The pairs can be found at poseidon .eur.nl/memorylab/Publications/StimuliNeighborProperty.pdf. The noun-property pairs were presented in short sentences, such as Een biet is vloeibaar (A beet is liquid; note that biet [beet] is a neighbor of bier [beer]). An additional, new set of 16 practice and 80 filler sentences was created with less specific properties. The unrelated control condition and counterbalanced list were constructed in the same way as in Experiment 1. All other aspects of the method were identical to that of Experiment 1.

\section{Results}

For each condition, the mean RT was calculated. The trimming procedure resulted in the removal of $1.1 \%$ of the RTs to correct responses. RTs for incorrect responses were also excluded. Table 2 shows the mean RTs and EPs. Analyses were done with relatedness as a within-participants factor and list version as a between-participants factor. Responses were slower to properties that were true for a neighbor of the target noun than to properties that were not true for any of the neighbors of the target noun $[F(1,52)=$

Table 2

Mean Reaction Times (RTs, in Milliseconds), Error Percentages (EPs), and Standard Errors (SEs) in the Property Verification Task of Experiment 2

\begin{tabular}{|c|c|c|c|c|}
\hline \multirow[b]{2}{*}{ Condition } & \multicolumn{2}{|c|}{ RT } & \multicolumn{2}{|c|}{ EP } \\
\hline & $M$ & $S E$ & $M$ & $S E$ \\
\hline Unrelated & 1,121 & 24 & 5.6 & 0.8 \\
\hline Related & 1,142 & 27 & 9.5 & 0.8 \\
\hline Neighbor interference effect & 21 & 10 & 3.9 & 0.7 \\
\hline
\end{tabular}

Note-In the related condition, the property was true for a neighbor of the target noun. In the unrelated condition, the property was not true for any neighbor of the target noun. 
$4.79, p<.05]$. In addition, participants made significantly more errors to properties that were true for a neighbor of the target noun than to properties that were not true for any of the neighbors of the target noun $[F(1,52)=33.33, p<$ $.001] .^{6}$ Thus, performance was better when the property was not true for a neighbor than when it was true for a neighbor of the target.

\section{EXPERIMENT 3}

The results of Experiments 1 and 2 are consistent with a cascaded view of word processing. In Experiment 3, we tested the prediction that neighbor interference effects would disappear with a long stimulus onset asynchrony (SOA) between the presentation of the noun and the presentation of the property. A long delay should allow the orthographic and semantic representations of the noun to be settled by the time the property is presented. Because orthographic processing and semantic processing of the noun are largely completed by the time the property is presented, the representation of the neighbor is no longer activated; hence, no neighbor interference effect would be observed.

Experiment 3 also allows us to rule out an alternative explanation of our results. The noun-property pairs that we used in the present study were designed in such a way that the sole difference between related and unrelated pairs was whether the property was or was not true of the target's neighbor. Despite this intention, there may have been another unintentional difference between the conditions in terms of relatedness between the property and the target itself. It may be that the related properties were more related to the target noun itself (rather than only to the neighbor of the noun) than were the unrelated properties. If indeed the effects in Experiments 1 and 2 were due to some unintentional differences in relatedness of the properties to the target nouns themselves, the effect should still be obtained in Experiment 3. If, on the other hand, the effects were due to the temporary activation of the semantic features of neighbors of the target noun (caused by cascaded processing), the neighbor interference effect should be absent in Experiment 3.7

\section{Method \\ Participants. Fifty-two students from Erasmus University Rot- terdam participated for partial course credit. All of the participants were native Dutch speakers. None had participated in Experiment 1 or Experiment 2. \\ Stimuli and Procedure. The same stimuli and procedure as in Experiment 2 were used, but with one important exception: The interval between the presentation of the first part of the sentence (which contained the noun, but not the property) and the presenta- tion of the complete sentence was increased to $1,000 \mathrm{msec}$.}

\section{Results}

For each condition, the mean RT was calculated. The trimming procedure resulted in the removal of $1.2 \%$ of the RTs to correct responses. RTs for incorrect responses were also excluded. Table 3 shows the mean RTs and EPs. Analyses were done with relatedness as a within-participants factor and list version as a between-participants factor.
Table 3

Mean Reaction Times (RTs, in Milliseconds), Error Percentages (EPs), and Standard Errors (SEs) in the Property Verification Task of Experiment 3

\begin{tabular}{lrrrrrr}
\hline \multirow{2}{*}{ Condition } & \multicolumn{3}{c}{$\mathrm{RT}$} & & \multicolumn{2}{c}{ EP } \\
\cline { 2 - 3 } \cline { 5 - 6 } & $M$ & $S E$ & & $M$ & $S E$ \\
\hline Unrelated & 790 & 21 & & 4.0 & 0.6 \\
Related & 787 & 21 & & 5.0 & 0.8 \\
Neighbor interference effect & -3 & 8 & & 1.0 & 0.8 \\
\hline
\end{tabular}

Note-In the related condition, the property was true for a neighbor of the target noun. In the unrelated condition, the property was not true for any neighbor of the target noun.

Responses to properties that were true for a neighbor of the target noun did not differ from responses to properties that were not true for any of the neighbors of the target noun [for RTs, $F(1,50)<1$; for EPs, $F(1,50)=1.66, p>$ $.20] .{ }^{8}$

Analyses of the combined data of Experiments 2 and 3 showed that the effect of delay interacted with the effect of relatedness [for RTs, $F(1,102)=3.95, p=.05$; for EPs, $F(1,102)=7.50, p<.01]$. Thus, the relatedness effect obtained when the delay between noun and property was $50 \mathrm{msec}$ disappeared when the delay was increased to $1,000 \mathrm{msec}$. These findings are consistent with the idea that the effect was due to the activation of the neighbor's semantic features.

\section{DISCUSSION}

In two experiments, we observed that participants were slower and less accurate in rejecting a false property when the property was true for an orthographic neighbor of the target than when the property was not true for an orthographic neighbor of the target. Experiment 3 showed that no such neighbor interference effect was obtained with a long $(1,000 \mathrm{msec}) \mathrm{SOA}$ between the presentation of the noun and the presentation of the property. These results provide evidence that semantic properties of orthographic neighbors are initially activated. This is consistent with the view that semantic information is activated before orthographic processing has been completed. The present findings are predicted by cascaded models of word processing, but are problematic for form-first models of word processing because the latter assume that a unique word has to be identified and selected for further processing before semantic information is activated.

Previous studies have also shown that semantic properties of neighbors affect target word processing (Pecher et al., 2005; Rodd, 2004). In these studies, however, participants made animacy (or animal) decisions to every target word presented in the experiment. The data from the present study provide a problem for the links model. It is important to note that links to semantic fields do not contain information about specific semantic properties of the word itself. They are only indices to groups of things that are similar. In the present study, rather than verifying whether a word is an exemplar of a taxonomic category, participants had to consider a different semantic property on each trial. Because links do not contain semantic prop- 
erties, they could not be used in the present study to bypass lexical candidates.

There is another crucial difference between the property verification task used in the present study and the category decision tasks used in previous studies. In category decision tasks, the same decision is made on every trial. Because the system knows beforehand what kind of decision needs to be made, the verification process can bypass candidates that do not have a link to the relevant semantic field. In the property verification task, however, a different semantic property needs to be verified on every trial. Because the system does not know the property before the processing of the target starts, information on the property cannot be used to bypass the verification process for lexical candidates. Rather, the present results show that many semantic properties of neighbors are activated during word processing.

In an attempt to salvage the strictly-form-first-modelplus-links-model explanation, K. I. Forster (personal communication, March 14, 2008) suggested an alternative mechanism to explain the present results. According to this account, when participants get to the property (e.g., growl, in A pear can growl), they activate objects that have the property. When one of those objects is a neighbor of the target word, this orthographic similarity is noticed and prompts the system to make a careful check of the target. This could explain slower responses when the property is true for a neighbor of the target. For several reasons, this does not strike us as a very attractive explanation of the present results. First, it is not clear why participants would resort to a rather cumbersome strategy wherein they generate the target on the basis of a property, even though the target is already provided in the sentence and, in fact, is presented prior to the property. Second, this targetgeneration strategy seems not only to be time consuming and effortful, but likely to fail, as well. In Experiment 2 in particular, the properties (e.g., can growl, is made of metal) were true for many objects. It is therefore highly improbable that participants were able to generate all of the possible words that possessed the given property.

In order to assess how likely it was that participants would come up with the neighbors of target words, we presented the properties to 10 new participants and asked them to verbally generate as many things as possible for which that property was true. They were given $10 \mathrm{sec}$ for each property. For the experimental properties, $6.9 \%$ of the first responses turned out to be the neighbor of a target word (mean latency, $3.74 \mathrm{sec}$ ), and $12.7 \%$ of the remaining responses turned out to be the neighbor of a target word (mean latency, $6.37 \mathrm{sec}$ ). For the filler properties (remember that these were true for the target), these numbers were $1.9 \%(4.32 \mathrm{sec})$ and $3.8 \%(6.90 \mathrm{sec})$, respectively. Thus, when the participants were explicitly asked to generate words for which the property was true, the likelihood of their generating the neighbor was quite low. In addition, the participants generated the target word for true trials in fewer than $6 \%$ of the trials, even though they had $10 \mathrm{sec}$ to do so. Because the use of a target-generation strategy would make sense only if the participants had been able to quickly and correctly generate the target word from the property, we consider it unlikely that the participants engaged in such a strategy.

It is also worth noting that the links model fails to explain some of the results we had previously obtained. In an animacy decision task, Pecher et al. (2005) found impaired performance for nonexemplar targets (e.g., mat, an inanimate word) that had an exemplar neighbor (e.g., cat, an animate word). This result is similar to the one obtained by Rodd (2004). In addition, however, Pecher et al. found enhanced performance for nonexemplar targets that had a nonexemplar neighbor. This result is inconsistent with the links model because it predicts that nonexemplar neighbors, which do not have a link to the relevant semantic field, do not affect target processing. In addition, the links model predicts that performance to exemplar targets will be negatively affected by the existence of exemplar neighbors. If one of the target's neighbors has a link to the relevant semantic field, performance will be slowed down because the neighbor enters the verification process. Contrary to this prediction, however, Pecher et al. obtained faster, rather than slower, responses to exemplar targets with an exemplar neighbor.

Cascaded models can explain our present results, as well as those obtained in previous studies. In cascaded models, the stimulus activates the orthographic patterns of the target word and of its neighbors. Activation cascades through the system, and, as a result, semantic features of the target word and also of its orthographic neighbors are included in the initial semantic representation. Hence, the semantic information that is activated corresponds to both the target and its neighbors. Subsequently, when the property is read, orthographic patterns corresponding to the property are activated, and these activate semantic information. If the property is read while the system is still trying to settle on the target word, the semantic activation due to the target's neighbor matches the semantic activation of the property more in the related condition than in the unrelated condition. In other words, because the neighbor is activated, some of the semantic features of the neighbor will also be activated. If the semantic features of the property are matched against all activated features (from the target noun and its neighbors), there will be more matching features when the property is true for the neighbor than when it is not true for the neighbor. The stronger the match, the harder it is to reject the property. Thus, relatedness leads to impaired responding.

In conclusion, our results show that properties of orthographic neighbors affect word processing. This effect is problematic for form-first models of word recognition, because these models predict that semantic information is activated before orthographic processing has been completed. Although the links model (Forster, 2006; Forster \& Hector, 2002) may be able to account for the findings obtained by Forster and Hector and by Rodd (2004), it cannot account for the present results, nor for some of the findings reported by Pecher et al. (2005). Our results can, however, be explained by cascaded models of visual word recognition. 


\section{AUTHOR NOTE}

This research was supported by a grant from the Netherlands Organization for Scientific Research (NWO) to D.P. We thank Saskia van Dantzig, Bruno Bocanegra, Mario de Jonge, Aljosja Jacobs, Wouter Platenburg, and Eric-Jan Wagenmakers for their assistance. We thank Derek Besner and Ken Forster for comments on an earlier version of the manuscript. Correspondence concerning this article should be addressed to D. Pecher, Psychology Department T12-33, Erasmus University, Postbus 1738, 3000 DR Rotterdam, The Netherlands (e-mail: pecher@fsw .eur.nl).

\section{REFERENCES}

Becker, S., Moscovitch, M., Behrmann, M., \& Joordens, S. (1997). Long-term semantic priming: A computational account and empirical evidence. Journal of Experimental Psychology: Learning, Memory, \& Cognition, 23, 1059-1082.

Boot, I., \& PeCher, D. (2008). Word recognition is affected by the meaning of orthographic neighbours: Evidence from semantic decision tasks. Language \& Cognitive Processes, 23, 375-393.

Bourassa, D. C., \& Besner, D. (1998). When do nonwords activate semantics? Implications for models of visual word recognition. Memory \& Cognition, 26, 61-74.

Bowers, J. S., Davis, C. J., \& Hanley, D. A. (2005). Automatic semantic activation of embedded words: Is there a "hat" in "that"? Journal of Memory \& Language, 52, 131-143.

Duñabeitia, J. A., Carreiras, M., \& Perea, M. (2008). Are coffee and toffee served in a cup? Ortho-phonologically mediated associative priming. Quarterly Journal of Experimental Psychology, 61, 18611872.

FORSTER, K. I. (2006). Early activation of category information in visual word recognition: More on the turple effect. The Mental Lexicon, 1, $35-58$.

Forster, K. I., \& Hector, J. (2002). Cascaded versus noncascaded models of lexical and semantic processing: The turple effect. Memory \& Cognition, 30, 1106-1117.

Hino, Y., Lupker, S. J., \& PeXman, P. M. (2002). Ambiguity and synonymy effects in lexical decision, naming, and semantic categorization tasks: Interactions between orthography, phonology, and semantics. Journal of Experimental Psychology: Learning, Memory, \& Cognition, 28, 686-713.

Masson, M. E. J. (1995). A distributed memory model of semantic priming. Journal of Experimental Psychology: Learning, Memory, \& Cognition, 21, 3-23.

McClelland, J. L., \& Rumelhart, D. E. (1981). An interactive activation model of context effects in letter perception: I. An account of basic findings. Psychological Review, 88, 375-407.

Pecher, D. (2001). Perception is a two-way junction: Feedback semantics in word recognition. Psychonomic Bulletin \& Review, 8, 545-551.

Pecher, D., Zeelenberg, R., \& Wagenmakers, E.-J. (2005). Enemies and friends in the neighborhood: Orthographic similarity effects in semantic categorization. Journal of Experimental Psychology: Learning, Memory, \& Cognition, 31, 121-128.

Pexman, P. M., \& LupKer, S. J. (1999). Ambiguity and visual word recognition: Can feedback explain both homophone and polysemy effects? Canadian Journal of Experimental Psychology, 53, 323-334.
Pexman, P. M., Lupker, S. J., \& Hino, Y. (2002). The impact of feedback semantics in visual word recognition: Number-of-features effects in lexical decision and naming tasks. Psychonomic Bulletin \& Review, 9, 542-549.

RAAIJMAKERS, J. G. W. (2003). A further look at the "language-as-fixedeffect fallacy." Canadian Journal of Experimental Psychology, 57, 141-151.

Raaijmakers, J. G. W., Schrijnemakers, J. M. C., \& Gremmen, F. (1999). How to deal with "the language-as-fixed-effect fallacy": Common misconceptions and alternative solutions. Journal of Memory \& Language, 41, 416-426.

RodD, J. M. (2004). When do leotards get their spots? Semantic activation of lexical neighbors in visual word recognition. Psychonomic Bulletin \& Review, 11, 434-439.

Stone, G. O., Vanhoy, M., \& Van Orden, G. C. (1997). Perception is a two-way street: Feedforward and feedback phonology in visual word recognition. Journal of Memory \& Language, 36, 337-359.

VAN Orden, G. C., \& Goldinger, S. D. (1994). Interdependence of form and function in cognitive systems explains perception of printed words. Journal of Experimental Psychology: Human Perception \& Performance, 20, 1269-1291.

Ziegler, J. C., Montant, M., \& Jacobs, A. M. (1997). The feedback consistency effect in lexical decision and naming. Journal of Memory \& Language, 37, 533-554.

\section{NOTES}

1. It should be noted that, of the articles cited here, only Becker et al. (1997) and Masson (1995) actually implemented a semantic level in their computer simulations, but the general principle holds for other models as well.

2. Note that in order to make an exemplar decision (e.g., decide that a lexical item refers to an animal), access to semantic information is still needed in this model after orthographic processing has been completed (i.e., after the verification process has determined that the lexical candidate matches the input).

3. The title of Rodd's article, "When Do Leotards Get Their Spots? Semantic Activation of Lexical Neighbors in Visual Word Recognition," may suggest that all kinds of semantic properties of orthographic neighbors are activated during orthographic processing. It should be noted, however, that this was not tested in her study, because an animal decision task was used.

4. We included list version in the analyses to reduce error variance (Raaijmakers, Schrijnemakers, \& Gremmen, 1999). Because it was not of theoretical interest, effects due to list version are not reported.

5 . In the main text, we report participant $\left(F_{1}\right)$ analyses. This is the correct analysis when a counterbalanced design is used (Raaijmakers, 2003; Raaijmakers et al., 1999). However, because some reviewers routinely insist on $F_{2}$ analyses, these are reported here. For RTs, $F_{2}(1,76)=5.79$, $p<.05$. For EPs, $F_{2}(1,76)=1.69, p>.1$.

6. For RTs, $F_{2}(1,76)<1$. For EPs, $F_{2}(1,76)=4.84, p<.05$.

7. We thank an anonymous reviewer for suggesting this experiment.

8. For RTs, $F_{2}(1,76)<1$. For EPs, $F_{2}(1,76)=1.43, p>.20$.

(Manuscript received March 28, 2008; revision accepted for publication January 27, 2009.) 